\title{
Balkanologie
}

Balkanologie Revue d'études pluridisciplinaires

Vol. IX, n' $1-2 \mid 2005$

Volume IX Numéro 1-2

\section{Shkëlzen ou Giannis ? Changement de prénom et stratégies identitaires, entre culture d'origine et migration}

Shkëlzen ou Giannis? First Name's Changes and Identity Strategies, between Homeland's Culture and Migration

\section{Georgia Kretsi}

\section{OpenEdition}

Journals

Édition électronique

URL : http://journals.openedition.org/balkanologie/583

DOI : 10.4000/balkanologie.583

ISSN : 1965-0582

Éditeur

Association française d'études sur les Balkans (Afebalk)

Édition imprimée

Date de publication : 1 décembre 2005

ISSN : 1279-7952

\section{Référence électronique}

Georgia Kretsi, « Shkëlzen ou Giannis ? Changement de prénom et stratégies identitaires, entre culture d'origine et migration », Balkanologie [En ligne], Vol. IX, n 1-2 | 2005, mis en ligne le 13 janvier 2010, consulté le 17 décembre 2020. URL : http://journals.openedition.org/balkanologie/583 ; DOI : https:// doi.org/10.4000/balkanologie.583 


\title{
SHKËLZEN OU GIANNIS ? CHANGEMENT DE PRÉNOM ET STRATÉGIES IDENTITAIRES, ENTRE CULTURE D'ORIGINE ET MIGRATION ${ }^{1}$
}

\author{
Georgia Kretsi*
}

" Dis-moi Giannis [un prénom grec, N.d.A.]. Je m’appelle comme ça maintenant. " " Et avant ? " " Giannis. Je m'appelais aussi comme ça avant ", répond l'Albanais d'origine Shkëlzen S. Les paroles de Giannis soulignent une césure que toutes les Albanaises et tous les Albanais ont connue dans leur itinéraire de vie, entre l'“aujourd'hui" d'après les changements politiques et le "hier" d'avant l'ouverture des frontières. Ils manifestent le souhait d'être des "Giannis" et le besoin de dissimuler le passé. Il y a de nombreuses raisons valables de vouloir dissimuler un prénom albanais en Grèce et de tout miser sur "aujourd'hui" et sur un nouveau prénom. Mais si l'on abandonne l'hypothèse que chaque être humain ne peut être identifié qu'avec un seul prénom, alors il faut reconsidérer l'apparente contradiction du dialogue cité précédemment. Ce changement de prénom apparemment indolore mérite toute notre attention².

La grande majorité des Albanaises et des Albanais qui immigrent en Grèce adopte un prénom grec-orthodoxe. Les Albanaises et les Albanais musulmans

\footnotetext{
${ }^{1}$ Une première version de cet article a été publiée en allemand dans Kaser (Karl), Richler (Robert), Schwandner-Sievers (Stephanie), Hg., Die weite Welt und das Dorf. Albanische Emigration am Ende des 20. Jahrhunderts, Wien : Böhlau, 2002. Traduction en français par Xavier Bougarel.

-Historian-Anthropologist, Associated Researcher at the Osteuropa-Institut, Freie Universität, Berlin. Contact : kretsi@gmx.de

${ }^{2}$ Je voudrais ici mentionner le fait que je me suis intéressee pendant ma recherche de terrain aux traditions liées au choix des prénoms. En ce qui concerne les noms de famille, ils sont stables depuis le début du XXème siècle dans les deux villages étudiès, Fterra et Çorraj, et sont transmis par les hommes du groupe de parenté (alb. : fis). Sans vouloir ignorer le rôle structurant des noms de famille pour les liens de parenté et le tissu social villageois, cette fonction particulière n'est pas déterminante pour les comportements en Grèce où les changements concernent en premier lieu les prénoms. Il n'y a de connotation religieuse que dans la transmission intergénérationnelle des prénoms. Une recherche complémentaire, qui prendrait en compte un plus grand nombre de lieux d'émigration albanais, pourrait fournir des résultats intéressants sur l'évolution des noms de famille et sur les éventuelles influences dues aux phénomènes migratoires.
} 
renient leur appartenance religieuse ${ }^{3}$ et se déclarent chrétiens orthodoxes. Les opinions publiques grecque et albanaise n'ignorent pas ces pratiques. Du côté grec, ce processus est considéré comme positif pour la préservation de l'homogénéité culturelle du pays. Du côté albanais, il s'agit d'une stratégie éprouvée pour s'intégrer dans un environnement nouveau de la manière la plus discrète possible. Pourtant, l'utilisation de différents prénoms et le traitement apparemment désinvolte du prénom reçu à la naissance apparaissent comme déroutants ou incompréhensibles aux yeux des observateurs extérieurs.

L'entrée dans un univers étranger déclenche une sorte de réaction en chaîne. Le changement de prénom et de religion doit être considéré comme une réaction au passage dans un univers nouveau, en particulier parce que l'“exode" albanais vers la Grèce (1990/1991) s'est réalisée "à travers champs" et que les gens étaient confrontés aux risques de la clandestinité. L'impression que les immigrantes et les immigrants de souche grecque sont mieux accueillis alors que les musulmans se heurtent à de la méfiance s'avère exacte dans bien des cas. C'est ainsi que de nombreux Albanaises et Albanais se sont faits baptiser, certains à la demande de leur employeur, d'autres afin de gagner à travers le parrainage les amitiés et les soutiens sociaux leur permettant de prendre pied dans la société grecque.

Le changement de prénom ne s'est toutefois pas limité à la vie quotidienne en contexte migratoire, mais a aussi été pratiqué en Albanie. La déliquescence de l'appareil administratif, la corruption de l'administration et la destruction d'une grande partie des registres civils qui ont accompagné la période des changements politiques en Albanie ont facilité ces changements de prénom légaux ou illégaux. Après les changements politiques s'est développée dans les institutions albanaises une vaste activité de falsification de documents officiels. La finalité de ces falsifications était, dans le cas des migrantes et des migrants vers la Grèce, de se présenter au consulat de Grèce avec de nouveaux documents et d'avoir ainsi une plus grande chance d'obtenir un visa. Car les chances d'obtention d'un visa augmentaient dès que l'on pouvait prouver son origine grecque et sa foi chrétienne orthodoxe. En Grèce également, le "passeport nord-épirote" 4 était utile. Le nouveau passeport représentait une

\footnotetext{
3 Par "appartenance religieuse", nous entendons ici uniquement l'affiliation à une tradition religieuse familiale, vu que la pratique religieuse elle-même a été interdite en Albanie par l'État communiste et athée.

${ }^{4}$ Au début, les membres de la minorité grecque ont obtenu des "passeports" (ou "cartes d'identité") spéciaux délivrés par l'organisation de la minorité grecque OMONIA, afin de pouvoir franchir la frontière. Plus tard, différentes versions de ces passeports ont été attribués par des associations basées en Albanie ou en Grèce (Syllogos Enossis Ellinon Voriou Ipirou [Association unifiée des Grecs d'Epire du nord], Vorioipirotikos Syllogos [Association nord-épirote], etc.) et qualifiés de "nord-épirotes". Le qualificatif de "nordépirote" est à rattacher à la vieille revendication irrédentiste selon laquelle le sud de l'Albanie représente en fait une partie de l'Épire grecque. Les " passeports n n'équivalent à un permis de séjour, mais facilitent implicitement la prolongation des visas et l'obtention d'un permis de travail en Grèce. Il est important de
} 
protection potentielle pour les migrants, dont une grande partie séjournait en Grèce de manière semi-clandestine et travaillait souvent au noir. S'ils pouvaient prouver leur origine grecque de manière suffisamment convaincante, il y avait une chance qu'ils échappent à l'expulsion.

Les pressions assimilationnistes auxquelles les migrantes et les migrants étaient et sont soumis en Grèce ont peu à peu suscité un intérêt scientifique croissant 5 . Pourtant, trop peu d'attention a jusqu'à présent été accordée à l'impact du changement de prénom décidé par les immigrés sur leurs choix et sur leurs vécus identitaires. La question se pose donc de savoir si l'adoption d'un prénom nouvellement "acquis" - au sens bureaucratique - va de pair avec l'apparition d'un "nouveau" mode d'identification sociale et/ou nationale, et comment ce "nouveau" mode d'identification doit ètre compris dans son rapport à l'"ancien".

\section{PRÉNOM ET MODES D'IDENTIFICATION}

Le facile changement de prénom chez les migrants s'explique par leur expérience de deux univers clairement inégaux. Les deux espaces sociaux en question symbolisent l'ancien et le nouveau. Par conséquent, l'ancien et le nouvel univers sont d'emblée associés à des expériences extrêmement divergentes. On pourrait supposer que le fossé existant entre le passé en Albanie et le présent en Grèce suscite une forte tension et une concurrence entre les deux prénoms et les deux registres identitaires. L'opinion des villageois et des villageois de Fterra sur le changement remet toutefois cette supposition en question :

noter que, alors que le terme "nord-ëpirote" n'était pas utilisè pendant la pėriode communiste, il est aujourd'hui revenu dans la langue albanaise. Sur la racialisation de la politique grecque d'immigration et les politiques de patronage ethnique, voir Chxistopoulos (Dimitris), I Ellada tis omogenias. Apo miterapatrida, kapitalistiki mitropoli (La Grèce de l'homogénéité : de la mère-patrie à la métropole capitaliste). $O$ Politis, (109), 2002 ; Kretsi (Georgia), " The Uses of Origin : Migration, Power-Struggle and Memory in southern Albania ", in King (Russell), Schwandner-Sievers (Stephanie), Mai (Nicola), eds., The New Albanian Migration, Brighton : Sussex Academic Press, 2004.

5 Un ensemble de stèréotypes nėgatifs et d'affirmations erronées sur " le " migrant albanais en Grèce conduit à une réaction en chaîne poussant les migrants dans l'“illégalité" et donc, potentiellernent, dans la "criminalité", et légitime dès lors leur exclusion sociale et leur exploitation. Sur la genèse du stéréotype de l'"Albanais criminel", voir Karydis (Vassilis), I eglimatikotita ton metanaston stin Ellada. Zitimata theorias ke adeglimatikis politikis (La criminalité des migrants en Grèce. Questions théoriques et politiques de lutte contre la criminalité), Athènes : Papazisis, 1996. Toutefois, les difficultés qu'ont connues les migrants albanais en Grèce durant la première période de migration (1990-1997) se sont amoindries depuis les régularisations de 1998 et des années suivantes. Sur les transformations et les enjeux complexes du processus migratoire, voir Marvakis (Athanasios), Parsanoglou (Dimitris), Pavlou (Miltos), eds, Metanastes stin Elladas (Les migrants en Grèce), Athens : Ellinika grammata, 2001. 
- A. B. : " Son nom ne lui a jamais plu [à son mari], il l'a fait changer auprès des services de l'état civil. Les noms ne viennent en fait que de l'État, c'est l'affaire de l'État. ” - C. D. : " Non, ce n'est pas difficile de changer mon nom. Mon mari aussi devra m'appeler autrement si je me fais baptiser. La nona [marraine] décidera du nom qu'elle souhaite pour moi. (...) Ce n'est pas un problème. Ce n'est qu'un nom, justement qu'un nom. "

- E. F. : " Mon ami [grec] voulait que je m'appelle Petro, il voulait ainsi renforcer notre amitié. Je l'ai donc fait très volontiers."

- G. H. : « Le nom n'a pas d'importance. Ce n'est jamais qu'une affaire de bureaucratie."

Ces citations donnent l'impression que, chez les personnes interrogées, le prénom est l'objet d'un mode de valorisation qui nous est étranger, et est considéré comme un élément interchangeable au cours de la vie. À une telle attitude s'oppose le point de vue qui entretient plutôt un lien familial ou religieux étroit avec le prénom, et qui se rencontre fréquemment dans l'espace chrétien. Dans cette conception, le prénom est inséparable de la personne, il est un marqueur identitaire qui ne peut être échangé.

Le caractère irremplaçable du nom personnel est plus prononcé dans les sociétés dans lesquelles le nom est inscrit dans le système administratif ou dans lesquelles les noms reflètent des liens sociaux au sein du groupe. Si ces conditions structurelles n'existent pas, alors il faut renoncer à l'idée de la pérennité du nom, c'est-à-dire à l'identification d'une personne avec un seul nom, et s'accoutumer à son usage multiple. De ce point de vue, le facile changement de prénom mentionné par nos informantes et nos informants albanais doit donc être considéré dans sa signification sociale actuelle.

Le prénom est avant tout un élément de l'identification personnelle. Par ailleurs, il peut lier l'individu à son groupe. Chaque prénom porte en lui un caractère identitaire intrinsèque ; toutefois, les rapports qu'un prénom établit avec ce groupe sont culturellement conditionnés. C'est pourquoi un aperçu des pratiques d'attribution des prénoms dans le domaine de la vie villageoise permet d'éclairer les interprétations culturelles possibles et de préciser le décalage supposé entre l'“ancien" et le "nouveau", la patrie d'origine et le pays d'accueil.

La recherche présentée ici s'appuyant sur des données recueillies sur le terrain, la dimension historique de l'attribution des prénoms ne peut être traitée ici. Une recherche sur les pratiques d'attribution des prénoms s'appuyant sur des bases de données historiques fournirait des connaissances riches d'enseignement ${ }^{6}$. Pour pouvoir mieux saisir l'attitude albanaise contemporaine

\footnotetext{
${ }^{6}$ Pour un premier aperçu sur les évolutions historiques des pratiques d'attribution des noms en Albanie, voir Kretsi (Georgia), “ Syghrones ke istorikes diastasis tis anthroponymikis simberiforas stin alvaniki kinonia " (Actual and Historical Dimensions of Anthroponymic Behavior in Albanian Society), Mnimon, (25), 2003.
} 
envers l'attribution des prénoms et relativiser "nos" propres représentations concernant cette pratique culturelle, je vais maintenant opposer les formes "grecque" et "albanaise" d'attribution des prénoms.

\section{L'ATTRIBUTION DES PRÉNOMS DANS UNE PERSPECTIVE COMPARATISTE : ALBANIE ET GRECE}

"Notre" propre regard sur l'utilisation des prénoms par les Albanaises et les Albanais, tout comme celui de la société grecque qui réagit à ce comportement, est en partie influencé par des attitudes culturelles elles-mêmes liées à une tradition administrative. Cette tradition est fondée sur l'intangibilité des noms. Si nous observons l'usage des noms dans d'autres sociétés faiblement organisées sur le plan administratif, nous constatons des phénomènes similaires, un rapport au nom similaire au cas albanais. Dans les sociètés dans lesquelles le nom n'est pas officiellement enregistré, sa manipulation privée est un phénomène courant?

Dans le cas des migrantes et des migrants albanais, ce constat s'applique au pays d'accueil dans lequel l'assignation identitaire des migrantes et des migrants ne joue pas, puisqu'ils n'y sont pas officiellement enregistrés. Ils peuvent donc pratiquement faire ce qu'ils veulent avec leur prénom. L'effondrement de l'État et de l'appareil administratif albanais a rendu encore plus facile ce genre de pratiques.

En Grèce, au contraire, le nom personnel est lié à l'administration, aux obligations envers l'État et à la possibilité d'exercer certains droits civiques. C'est pourquoi les pratiques des Albanaises et des Albanais sont souvent perçues comme malhonnêtes et illégales. Pour ces derniers, toutefois, le facteur décisif est un rapport brouillé aux deux États albanais et grec. Depuis les changements politiques, les institutions étatiques ne sont pratiquement plus prises en considération par les citoyennes et les citoyens albanais - et dans bien des cas elles n'existent plus. Plus généralement, l'État n'a pas été en mesure d'intégrer les individus. Il a donc non seulement perdu le contrôle sur ses citoyennes et citoyens - à l'intérieur du pays comme à l'étranger - mais a modelé leur rapport général aux institutions publiques. À leurs yeux, l'État incarne plutôt une menace pour leurs propres intérêts personnels. Il est donc de leur intérét, en ce qui concerne par exemple la recherche d'un emploi, de choisir eux-

\footnotetext{
7 Dans différents contextes culturels, on observe que les noms personnels peuvent ètre modifiés plu. sieurs fois au cours d'une vie, et en particulier au moment d'importants rites de passage. Voir par exemple Zawawi (Sharifa M.), African Muslim Names : Images and Identities, Trenton, NJ : Africa World Press, 1998, pp. 85-107 et, pour l'utilisation de "noms multiples", Hastings (James), ed., Encyclopedia of Religion and Ethics, vol. IX, Edinburgh : T. \& T. Clark, 1971, pp. 130-131, 165.
} 
mêmes leur prénom, indépendamment des cadres juridiques. Sur la base de ce constat, les propos de A.B. et G.H. deviennent compréhensibles : le nom est seulement "une affaire de bureaucratie", ou est attribué "par l'État". Mais, du fait que l'État et la bureaucratie ont perdu de leur crédibilité, les prénoms ont aussi perdu de leur importance. Dans ce contexte, le changement de prénom révèle un manque d'identification à l'État. Ce qui est décisif dans la perception de soi n'est pas l'ancrage dans chacun des deux États, mais la flexibilité et la capacité de leur échapper.

On peut considérer comme très probable le fait que, lors de leur future légalisation et intégration dans le système social grec, les migrants albanais déclareront et conserveront leur prénom. Les prénoms traditionnels perdront de leur importance et les nouveaux accompagneront le processus d'identification à l'État grec. La deuxième génération portera évidemment des prénoms chrétiens orthodoxes, et donc "grecs". Sur le plan administratif, il ne sera alors plus possible de distinguer la minorité d'origine albanaise en Grèce de la minorité grecque de citoyenneté albanaise.

"Vous êtes coupables de ne pas savoir qui vous êtes ! " (dit le Grec à l'Albanais, N.d.A.). " Ça ne va pas que dans une famille, un enfant s'appelle Tachir [prénom musulman, N.d.A.] et l'autre Kitsio [prénom chrétien, N.d.A.] ! (...) Ça ne va pas non plus que, pendant le ramadan, la femme mange le matin et les hommes pendant la nuit ! ${ }^{8}$ Dans ce texte littéraire de l'auteur grec Gourogiannis, un conflit culturel tout à fait réel devient explicite. Ce qui apparaît comme de l'inconséquence à des yeux "étrangers", parce qu'ils perçoivent justement le prénom comme un marqueur identitaire et une partie essentielle d'une tradition religieuse et culturelle, est une attitude normale dans la société albanaise. La coexistence de prénoms chrétiens et musulmans apparait dans ce texte comme déconcertante. Mais ce texte lui-même, qui apparait comme de la fétichisation aux yeux de l'autre partie, attribue aux prénoms une essence culturelle s'exprimant peu ou prou au travers de la religion.

Pour une grande partie de la société grecque, le prénom est considéré comme intangible. La culture de la transmission intergénérationnelle des prénoms est particulièrement présente. Le transmission du prénom des grandsparents aux petits-enfants symbolise la perpétuation des âmes et l'insertion familiale. La forte valorisation de la transmission intergénérationnelle dans la culture grecque de l'attribution des prénoms souligne le rôle des réseaux familiaux et des liens intergénérationnels, et ce en dépit de l'urbanisation. Le rapport entre le prénom, les rapports d'héritage et les obligations familiales connaît une multitude de variations locales qui ne peuvent être présentées

${ }^{8}$ Gourogiannis (Vasilis), To assimoharto anthizi (La fleur de papier d'argent éclot), Athènes : Kastaniotis, 1996, pp. 38-39. 
ici 9 . De manière générale, toutefois, il est possible de constater que dans le christianisme, le prénom entretient un rapport étroit avec la foi. Il repose sur un lien spirituel avec le saint correspondant, place la personne ainsi nommée sous sa protection et la prépare ainsi pour la vie après la mort. De plus, le prénom montre l'insertion au sein de la communauté chrétienne orthodoxe, et devient ainsi pour l'individu un marqueur identitaire indispensable. Le prénom est attribué dès le plus jeune âge, et il obtient sa consécration à l'église à travers le baptême. Ce rite de passage vaut pour le restant de la vie, et n'est pas complété ou élargi par un autre sacrement chez les chrétiens orthodoxes, comme cela est le cas chez les protestants avec la confirmation ou chez les catholiques avec la communion.

L'appartenance religieuse a joué un rôle significatif dans le processus de construction nationale des deux peuples balkaniques. À l'époque de l'Empire ottoman (du XVème au XXème siècle), l'appartenance "ethnique" était définie par la compétence administrative des millets (chrétien, juif, arménien). Du fait que les Grecs occupaient des positions très élevées dans la hiérarchie du millet chrétien orthodoxe, la chrétienté orthodoxe dans son ensemble était parfois qualifiée de "Grecs"10. Ce système ayant attribué une signification décisive, non pas à la langue mais à la religion, l'insertion des Albanaises et Albanais orthodoxes dans la nation albanaise n'a pu se faire que très progressivement. La participation de nombreux Albanais orthodoxes (appelés Arvanites ou Albanophones) au mouvement de libération nationale grec témoigne de l'importance de l'appartenance religieuse pour les affiliations ethniques de l'époque $^{11}$.

Du point de vue idéologique, le nationalisme grec s'est autant nourri de la religion que de l'héritage imaginaire de l'Antiquité. À cette tendance qu'ont les Grecs de percevoir leur identité culturelle comme enracinée dans l'histoire, le mouvement national albanais ne peut opposer qu'une histoire courte, même si l'hypothétique origine illyrienne des Albanais permet de renvoyer à une

\footnotetext{
9 Plusieurs travaux sur la société grecque orthodoxe ont souligné la valeur symbolique et le rôle socioéconomique de la transmission intergénérationnelle du nom au sein du groupe familial. Voir entre autres Hirshon (Renée), Heirs of the Greek Catastrophe. The Social Life of Asia Minor Refugees in Piraeus, Oxford : Clarendon, 1989, pp. 203-206 ; Kenna (Margaret E.), « Houses, Fields, and Graves : Property and Ritual Obligation on a Greek Island ", Ethnology. An International Journal of Cultural and Social Anthropology, 15 (1), 1976 ; Vernier (Bernard), " Putting Kin and Kinship to Good Use. The Circulation of Goods, Labour, and Name on Karpathos (Greece) ", in Medick (Hans), Sabean (David W.), eds., Interest and Emotion : Essays on the Study of Family and Kinship, Cambridge : Cambridge University Press, 1984.
}

${ }^{10}$ Karpat (Kemal H.), " Millets and Nationality : The Roots of Incongruity of Nation and State in the PostOttoman Era n, in Braude (Benjamin), Lewis (Bernard), eds., Christians and Jews in the Ottoman Empire, New York : Holmes \& Meier, 1982, pp. 146,160.

${ }^{11}$ Irmscher (Johannes), " Die Albaner bei der Gründung des Griechischen Staates ", Studia Albanica, 2, 1964. Sur la religion comme élément constitutif de l'identité nationale, voir Skopetea (Elli), To " Protypo Vasilio " ke i Megali Idea. Opsis tou ethnikou provlimatos stin Ellada (1830-1880) (Le " Royaume idéal " et la Grande idée. Aspects du problème national en Grèce, 1830-1880), Athènes : Polytypo, 1988, pp. 119-135. 
profondeur historique considérable. Le mouvement national albanais, porté par des intellectuels albanais chrétiens et musulmans et érigeant la langue albanaise et l'origine illyrienne comme marqueurs identitaires communs, ne s'est développé qu'à la fin du XIX ${ }^{\text {ème }}$ siècle. Les différences de confessions et d'intérêts ont retardé le développement d'une idéologie stato-nationale. Les différentes religions sont très longtemps restées dans une relation symbiotique. Le souvenir d'un passé chrétien commun et l'appartenance à des ordres soufis hétérodoxes ont contribué à ce que la religion ne prenne pas des formes fortement dogmatiques chez les Albanais ${ }^{12}$.

Les effets de cette symbiose sur l'attribution des prénoms se reflètent dans les constellations de prénoms que l'on retrouve au sein de certaines familles albanaises ${ }^{13}$. Le prénom ne lie pas en premier lieu la personne à une communauté religieuse.

Pendant la période communiste, le lien déjà faible entre État et religion a été complètement supprimé du fait de la proclamation de l'athéisme comme idéologie officielle. L'affirmation dans un environnement communiste de " l'albanité comme religion de chaque Albanais " souligne le contraste entre les politiques nationales albanaise et grecque. Alors que, dans le cas grec, les constructions religieuse et nationale se renforcent mutuellement, dans le cas albanais, la nation et l'idéologie communiste se substituent à l'appartenance religieuse pour rendre l'État albanais homogène. La campagne d'albanisation qui s'est intensifiée à partir des années 1960 s'est appuyée sur une rupture avec les traditions. La jeune génération a eu pour mission de rompre avec les structures familiales patriarcales et les religions, perçues comme autant d'obstacles à la modernisation. Cette campagne a abouti à la révolution culturelle albanaise de 1967, calquée sur le modèle chinois, au cours de laquelle l'Albanie fut proclamée " premier État athée du monde " ${ }^{14}$.

${ }^{12}$ Sur les "mémoires" d'un passé chrétien commun, voir également Liolin (Arthur E.), "The Nature of Faith in Albania : Toward the $21^{\text {st }}$ Century ", East European Quaterly, 31 (2), 1997. Sur les ordres soufis, voir Clayer (Nathalie), " Der Bektaschi-Orden in Albanien ", in Daum (Werner), ed., Albanien zwischen Kreuz und Halbmond, Munich : Staatliches Museum für Völkerkunde, 1998 ; Elsie (Robert), A Dictionary of Albanian Religion, Mythology, and Folk Culture, London : Hurst, 2001. Sur le syncrétisme religieux an Albanie, voir Bartl (Peter), " Kryptochristentum und Formen des religiösen Synkretismus in Albanien ", Grazer und Münchener Balkanologische Studien, 2, 1967; Stadtmüller (Georg), “ Die Islamisierung bei den Albanern ", Jahrbücher für Geschichte Osteuropas, 3, 1955.

${ }^{13}$ Ainsi, les enfants de la famille D. à Fterra s'appellent Sokrati, Qemilë, Hamisë, Vera, Fotis, Mjaftone. Les prénoms grecs antiques, chrétiens et musulmans reflètent l'environnement dans lequel vit cette famille.

14 D'après Peter Bartl, plus de 2000 mosquées, tekkes, églises et monastères furent fermés dans ce contexte (Bartl (Peter), "Religionsgemeinschaften und Kirchen ", in Grothusen (Klaus-Detlev), Hg., Südosteuropa - Handbuch, Band VII (Albanien), Göttingen, 1993, pp. 608-6og). Sur les formes et les objectifs de cette "révolution culturelle". Voir également le chapitre " Lëvizja kundër fesë dhe zakoneve prapanike " (Le mouvement contre la religion et les traditions arriérées) dans Akademia e Shkencave e RPS të Shqipërisë, Historia e Shqipërisë (Histoire de l'Albanie), vol. IV, Tiranë : Akademia e Shkencave, 1983, pp., 337-339 ; ainsi que Pano (Nicholas C.), "The Albanian Cultural Revolution ", Problems of Communism, 23 
Dans ce processus d'albanisation, les prénoms ont joué un rôle important. Des prénoms albanais (prénoms d'origine illyrienne et autres prénoms albanophones) ont été promus par les autorités. Les prénoms religieux et autres prénoms dits "ètrangers" n'ont plus été tolérés ou, du moins, ont été dévalorisés dans le cadre de la politique d'albanisation. Des listes de prénoms présélectionnés ont été accrochées dans les services de l'état civil. Le refus de choisir un prénom non-religieux et albanais pouvait dans certaines circonstances ètre considéré comme une attaque contre l'État. Pour la première fois dans l'histoire albanaise, une tentative avait lieu de lier le prénom à l'identité nationale ${ }^{15}$.

Cette politique est aujourd'hui perçue par une partie des personnes interrogées comme une page sombre de l'histoire albanaise. Monsieur I. J. estime que "l'Albanie a été privée d'une partie [de sa culture, N.d.A.]. Un jeune, par exemple, même s'il a un haut niveau scolaire (...), ne sait pas comment se comporter. Mais si tu vas à l'église, tu l'apprends (...). La religion influence la culture, le comportement ". Pour l'autre partie des personnes interrogées, pour lesquels le socialisme a joué un rôle crucial, la politique du Parti communiste est considérée comme une occasion unique dans l'histoire albanaise de favoriser l'expression du sentiment national : " les autres prénoms étaient turcs, ceux-là sont à nous, ils sont albanais ".

Aux yeux des Grecs, I'histoire albanaise apparait comme récente et insignifiante, en opposition aux prétentions universelles de leur propre histoire. L'apparence légèreté dans les changements de prénom et de religion est interprétée comme une "absence de tradition", bien qu'elle justifie dans le même temps l'acculturation des Albanaises et des Albanais. En effet, un prénom chrétien orthodoxe constitue pour de nombreux employeurs et bailleurs grecs une garantie palpable de l'honnèteté de la personne employée ; au contraire, un prénom musulman a souvent une connotation d'étrangeté, de malignité et de malhonnêteté.

(4), July 1974 ; Grulich (Rudolf), " Albanien - seit 20 Jahren das erste atheistische Land der Welt ". Südosteuropa, 36 (11-12), 1987.

15 Après 1967, des décrets furent même publiés concernant les responsabilités et les obligations des organes administratifs quant à l'attribution de noms aux bàtiments, aux villes et aux institutions. Les décrets concernant les norns furent rédigés conformément aux nouveaux critères moraux de la "révolution culturelle" (voir Gazeta zyrtare, 14/02/67, décret $n^{\circ} 181$ et $01 / 07 / 69$, décret $n^{\circ} 201$ ). En 1975, un décret sur la modification des " prénoms et noms de familles inappropriés " fut même publié, légitimant la modification des prénoms et noms de famille ne correspondant pas aux orientations " politiques, idéologiques et morales saines n du régime (voir Gazeta zyrtare, $23 / 09 / 75$, décret $n^{\circ} 5339$ ). La portée de la politique communiste des prénoms peut ètre illustrée par cette statistique des services de l'état civil de Shkodër que rapporte Jusuf Shpuza, seion laquelle $47 \%$ des nouveaux-nés portaient un prénom dit "illyro-albanais" en 1967. et $100 \%$ trois ans plus tard. Shpuza (Jusuf), Vëzhgim për emrat familjarë të shqiptarëve (Considérations sur les norns de famille des Albanais), Shkodra : Idromeno, 1998, p. 150. 


\section{FTERRA ET ÇORRAJ: LES PRÉNOMS AU PAYS ET DANS L'ÉMIGRATION}

Notre informant M. D. vit depuis trois ans en Grèce. Il se déclare Épirote du nord, sa femme a changé de prénom et prévoit de se faire baptiser. Il ferait n'importe quoi pour se débarrasser du sentiment de " ne pas pouvoir sortir dans la rue " en Grèce. En principe, cependant, il ne veut changer ni de prénom, ni de religion. Sa femme est d'un autre avis. Elle est devenue une chrétienne convaincue et voit dans le baptême un pont vers leur nouvel environnement.

La fille de N. O. a émigré en Grèce. Là-bas, elle s'est faite baptiser et a échangé son prénom Donika pour celui de Panagjota ${ }^{16}$. De même, les filles de P. Q., aujourd'hui en Grèce, ne s'appellent plus Çapare, Valjet et Muside, mais Antonetta, Violetta et Maria. Leurs parents n'expriment aucun mécontentement particulier quant à ce changement. Ils essayent eux-mêmes de légitimer ces nouveaux prénoms en les utilisant.

Vilson est en Grèce depuis 1992 et s'appelle maintenant Vassil. Il n'est pas baptisé, mais indique qu'il apprécie beaucoup la foi orthodoxe et qu'il se rend souvent à l'église. Sa femme a toujours été chrétienne et n'a pas changé de prénom. Son fils s'appelait Admirim ; depuis qu'il est en Grèce, il s'appelle Aristoteles. Vilson interprète ce changement de prénom comme une "obligation" envers la nouvelle culture du pays d'accueil. En outre, leurs nouveaux prénoms sonnent comme leurs prénoms albanais : "Cela ne fait aucune différence, Vilson ou Vassil, Admirim ou Aristoteles".

La raison pour laquelle, selon eux, le changement de prénom va de pair avec l'incorporation dans la nouvelle société, n'est pas développée d'avantage. La plupart de nos informantes et de nos informants reprennent l'explication courante que les Grecques et les Grecs tout autant que les migrantes et les migrants avancent comme un propos idéologiquement neutre : "Ils ne peuvent (...) absolument pas prononcer les prénoms que nous avons. Ils ont les prénoms de l'église. (...) Les Grecs ne peuvent absolument pas les prononcer. "

Laissons-nous ici aussi guider par deux approches identitaires différentes : " Je m'appelle Giannis et je suis Voreiopiliouriot [17] ", dit sur un ton ironique un adolescent du village voisin de Piliouri, qui a déjà l'expérience de l'émigration. Madame C. D. dit quant à elle : "Bien sûr, si je me fais baptiser et que je change mon nom, qu'est-ce que je serai sinon une Grecque, pas vrai ? ".

L'adolescent s'observe lui-même et observe les jeux identitaires en Grèce avec sarcasme. Le comportement de la Grèce, mais aussi son propre comporte-

\footnotetext{
${ }^{16}$ Tous les prénoms qui apparaissent par la suite comme de "nouveaux" prénoms des migrants en Grèce appartiennent au répertoire des prénoms grecs typiques. C'est pourquoi ce point ne sera pas explicité de nouveau dans le texte.
}

${ }^{17}$ Néologisme formé à partir de Voreioepirot (Épirote du nord) et du nom du village Piliouri. 
ment envers la signification des prénoms est tourné en dérision. On ne peut pas rejeter l'hypothèse que l'adolescent tire une certaine fierté de son habileté à jouer de la situation. Mais il fait toutefois indirectement allusion à la contradiction qu'il y a à être en même temps de Piliouri et de Grèce.

Madame C. D., qui vivait en Grèce au moment de l'entretien, souhaite au contraire clarifier la situation. Pour elle, être Grecque signifie être intégrée socialement, ne pas se différencier des autres. Elle espère accéder à l'égalité des droits au sein de la société grecque à travers le changement de prénom et le baptême. Elle n'attribue pas au terme "Grecque" une signification ethnique, mais sociale. En outre, elle-même n'exclue pas une autre appartenance nationale : " Eh, Grecque et Albanaise en même temps. Je n'ai pas de problème avec ça. Qu'y pouvons-nous ? Après tout, nous avons émigré ici ! " Elle ne prévoit pas de s'installer pour toujours en Grèce et veut rentrer en Albanie dans cinq ans. L'appartenance nationale et religieuse ne doit être considérée ici que comme une adaptation aux circonstances extérieures.

Ici, ce sont en premier lieu les circonstances rencontrées en Grèce qui fournissent un modèle explicatif évident : "Mes enfants doivent être Grecs, je ne veux plus être poursuivie ! " (Madame C.D.) ; "Si je disais que je m'appelle Shefket, je ne trouverais pas de travail. (...) On ne donnera jamais du travail à une femme avec un nom musulman, afin d'éviter qu'elle rentre dans la maison de quelqu'un ou qu'elle en reçoive la clef. " (Monsieur G. H.)

Nous avons évoqué dans le précédent passage l'attitude envers les nonchrétiens, c'est-à-dire envers les musulmans. Mais comment les Albanais interprètent-ils l'attitude des Grecs à leur égard, qui les stigmatise comme "dangereux" et "criminels"18 ? Quelles stratégies de distanciation et d'invention identitaires mettent-ils en œuvre?

Parmi nos interlocutrices et nos interlocuteurs à Fterra, de nombreuses voix s'expriment de façon critique : " Les Grecs sont des racistes, (...) ils ne peuvent pas prononcer nos noms, je ne sais pas. Peut-être qu'ils ne le veulent pas. (...) Ils sont en conflit avec la Turquie. (...) Quand ils entendent un nom musulman, ils te considèrent aussitôt comme un Turc. " (Monsieur I. J.)

Il y a aussi des voix qui voit dans l'"albanophobie" de l'opinion publique grecque la conséquence des mauvais comportements des migrantes et des migrants albanais en Grèce : " Nous ne nous sommes pas bien comportés, alors que les Grecs sont très humains, oui, très humains. " (Madame T. O..). Parfois, les migrantes et les migrants partagent la vision stéréotypée sur eux-mêmes qui s'est développée en Grèce. Ils ont sûrement « beaucoup volé, commis de nombreux meurtres, se sont mal comportés ". " Nous sommes nous-mêmes coupables ", dit notre informant E. D. de Çorraj, " en fait, il faudrait expulser de Grèce tous les Albanais!" 
Si l'on regarde l'itinéraire migratoire de chacune et de chacun, on s'aperçoit que le passage dans l'illégalité a pratiquement commencé dès les procédures de demande de visa ou dès la fuite. Il est donc légitime pour eux de prendre leur distance à l'égard du groupe albanais et de devenir chrétien orthodoxe ou "Épirotes du nord".

Une illustration de ce phénomène pourrait être la réponse de Monsieur I. J. à la question de savoir pourquoi il n'utilise pas seulement un nouveau prénom usuel, mais a aussi fait modifié son passeport : "j'ai acheté une voiture, j'ai mis de l'argent de côté. J'ai beaucoup de choses. Donc, j'ai acheté une voiture. J'ai en Grèce une voiture avec des plaques d'immatriculation grecques. Je veux être dans les règles, je ne veux pas vivre avec deux noms ". Car il prévoit de " rester longtemps et non de rentrer après cinq ans ". Si l'on voit ses plaques d'immatriculation en Grèce, chacun pensera qu'il est Grec. Cela lui assure une certaine sécurité car, en tant qu'Albanais, " on a toujours de gros ennuis avec la police ". Dans un contexte marqué par l'insécurité, l'assignation identitaire par le nouvel environnement social devient le critère de son propre mode d'identification. La décision de vouloir rester en Grèce signifie dans le même temps par celle de s'approprier l'ordre social grec.

Un tel changement de mode d'identification est d'une part une condition imposée par le nouvel environnement extérieur. Mais ce choix identitaire résulte aussi de choix sociaux et idéologiques. Les aspirations sociales dominantes en Grèce gagnent en importance au détriment de celles présentes en Albanie, car elles permettent entre autres un accès à l'"occidentalisation" et à la "modernité".

C'est dans le contexte de ces diverses aspirations que se situe l'usage des prénoms et de la religion. Beaucoup considèrent que le baptême n'est qu'un acte public : " je reste quand même musulmane " (Z. B.). " Même si je me faisais baptiser, parce que j'y étais forcée comme l'ont été mes sœurs, je resterais quand même une musulmane car je suis croyante ", dit Madame K. O. Monsieur R. S. a modifié son prénom en Grèce et se dit également prêt à se faire baptiser si son employeur le lui demande : " comme ça, par amitié, pour le qejf [plaisir], pas par contrainte, puisque j'ai de toute façon une autre religion ". Dans ces conceptions, la foi musulmane et le baptême chrétien sont tout à fait compatibles.

D'autres ne voient pas de différence essentielle entre les religions : " il y a autant de religions que de partis ", estime Madame T. Q. " Nous allons régulièrement à l'église. (...) Il n'y a qu'un seul Seigneur. Indépendamment de la religion. " (I. J.) Cette attitude envers le divin, qui dépasse les clivages confessionnels et "nous" est étrangère, semble faciliter l'adoption de la religion chrétienne. Outre le syncrétisme historique déjà mentionné, les conséquences de la politique athéiste communiste ne doivent pas être sous-estimées.

En outre, la religion prend une autre signification culturelle à travers les 
nouvelles expériences faites à l'étranger :

Quand nous circulons dans la rue, nous ne montrons aucun intérêt pour nos églises (...) Eux [les Grecs, N.d.A.] lãchent le volant et touchent leur croix, parce qu'ils ont du respect pour la religion et parce que leur culture l'exige. De même, en ce qui concerne les cimetières, (...) nous respectons beaucoup les chrétiens parce que nous n'allons pas au cimetière, (...) nous oublions vite, dit I. J.

En comparaison à l'Albanie nouvelle, les chrétiens en Grèce semblent se conformer plus scrupuleusement aux rituels et être plus soucieux d'honorer leurs ancêtres. La piété qui transparaît dans ces propos n'est pas feinte. La culture étrangère est imprégnée d'un sentiment religieux qui laisse une forte impression chez beaucoup de migrantes et de migrants albanais.

Dans le même temps, toutefois, les Albanaises et les Albanais réagissent différemment aux tensions qui existent entre la conception grecque et la conception albanaise de la culture. Ils prennent leurs distances par rapport au fanatisme religieux et nationaliste qu'ils perçoivent dans la société grecque. Par contre, l'ouverture envers le reste du monde et particulièrement envers la Grèce est souvent présenté comme une partie intégrante de la culture de l'Albanie du sud : " (...) nos ancêtres allaient au marché à Ioannina, depuis toujours, qu'ils soient musulmans ou chrétiens. Ils sont partis faire le kurbet [migration saisonnière - N.d.T.] pour travailler en Grèce et en Turquie, comme nous le faisons aujourd'hui. Ils savaient ce qu'est la culture ", dit R. S.

Finalement, cette tension aboutit à des constructions identitaires parallèles. La culture et la religion sont interprétées comme des capitaux auxquels il est possible de participer, sans toutefois devoir s'y identifier complètement. L'appartenance au groupe albanais peut tout à fait s'accorder avec d'autres sentiments d'appartenance. Un prénom grec (chrétien) n'implique pas une identité non-albanaise (non-musulmane). Au contraire, il signale une polytaxis $^{19}$, un déplacement parmi plusieurs modes d'identification possibles.

Cette tactique, à savoir " le passage rapide d'un cadre de référence à l'autre ${ }^{20}$ dans le nouvel environnement, n'est pas un phénomène isolé ne concernant que les Albanaises et les Albanais. De nombreux phénomènes similaires existent, par exemple, chez les Pomaques de Bulgarie ${ }^{21}$, les Athigani (Roms) du nord de la Grèce ${ }^{22}$ ou les migrants aux États-Unis. Ce type de comportement est

19 Par ce terme, l'anthropologue Georg Elwert désigne une multiplicité d'ordres s'interpénétrant de manière implicite. Voir Elwert (Georg), "Switching of We-Group Identities : The Alevis as a Case among Many Others ", in Kehl-Bodrogi (Krisztina), ed., Syncretistic Religious Communities in the Near East, Leiden : Brill, 1997 .

${ }^{20}$ Elwert (Georg), art.cit., p. 67.

${ }^{21}$ Karagiannis (Evangelos), Zur Ethnizität der Pomaken Bulgariens, Münster : Lit Verlag, 1998, pp. 86-130.

${ }^{22}$ Trubeta (Sevasti), Die Konstitution von Minderheiten und die Ethnisierung sozialer und politischer 
particulièrement prononcé au sein de groupes marginalisés qui ont intérêt à être intégrés dans la société majoritaire et cherchent ainsi à échapper à l'exclusion dont ils sont l'objet.

La question se pose de savoir si le changement de prénom peut être interprété comme une négation du groupe d'origine et une rupture avec ce dernier. Quelle valeur et quelle signification symbolique ont les prénoms pour les communautés de Fterra et Çorraj?

En ce qui concerne la culture anthroponymique au pays, il existe une liberté manifeste à Fterra et, dans une moindre mesure, à Çorraj, pour le choix du prénom des enfants - du moins au sein des jeunes générations. La politique communiste des prénoms a trouvé là un écho favorable, dans la mesure où la plupart des personnes interrogées voient dans les prénoms albanais un renouvellement et même un enrichissement du patrimoine anthroponymique.

Pendant notre séjour à Fterra, les généalogies de 30 foyers environ ont été relevées ${ }^{23}$. Les noms de famille de toutes les personnes habitant le village au moment de l'enquête ont été intégrés dans ce relevé. Il n'y a pas d'éléments suggérant l'attribution récente du prénom ou d'une partie du prénom d'un grand-parent ou d'un autre parent à un nouveau-né. Dans les généalogies, 29 prénoms apparaissent plusieurs fois, mais dans quatre cas seulement il s'agissait de transmission du prénom du grand-père ou de l'arrière-grand-père. Les autres occurrences multiples concernaient des personnes sans lien de parenté. À la question de savoir si les ancêtres étaient pris en considération au moment de l'attribution des prénoms, la réponse à Fterra était généralement non. Seuls les plus anciens se souvenaient d'une telle pratique. Les généalogies suggèrent cependant que le principe de recours à une même initiale est en usage, par exemple dans le cas de la fratrie Axinia, Antika, Aoreta et Afo.

Les prénoms les plus fréquents parmi les habitants âgés de Fterriot sont Murat, Ardi/Ardit, Mehmet, Lefteri, Marianna, Idriz, Idrizia et Ahmet. La plupart ont une origine musulmane ${ }^{24}$. Mais il y a aussi des prénoms tels que Lefteri (liberté) qui ont une origine grecque. Après 1970 apparaît un changement manifeste dans le choix des prénoms. Certains termes populaires de la langue albanaise se retrouvent plus fréquemment dans le répertoire des pré-

Konflikte. Eine Untersuchung am Beispiel der im griechischen Thrakien ansässigen " Moslemischen Minderheit ", Frankfurt am Main : Peter Lang, 1999.

23 Il faut souligner ici le fait que la mémoire généalogique de la plupart des personnes interrogées s'étend jusqu'à la troisième génération. C'est pourquoi il est difficile de donner des précisions sur les modèles historiques d'attribution des prénoms. Concernant les réactions contemporaines vis-à-vis des fréquents changements de prénom dans l'émigration, il est toutefois important d'identifier ce qui conserve encore une valeur sociale et symbolique aux yeux des habitantes et des habitants.

24 Mehmet et Ahmet sont des noms du Prophète. Idriz est dérivé de Enoch, un des 25 prophètes de la tradition juive. Ard vient aussi de la tradition juive et Marianna a été repris de la tradition chrétienne (Mirjam/Maria). 
noms, tels que Astrit (héros), Linda (naissance), Gëzim (joie), Flamur (drapeau). Les prénoms religieux passent peu à peu au second plan.

Les 33 cas relevés dans le village majoritairement orthodoxe de Çorraj indiquent une pratique de l'attribution des prénoms largement différente. Dans ce cas, on relève 78 occurrences multiples d'un prénom, qui reprennent en principe le prénom du grand-père paternel, mais aussi celui de la grand-mère paternelle. Les prénoms les plus fréquents sont Koste / Koco, Kater, Janni / Jannaqi, Dimiter / Dimitraqi, Maria / Marianna, Sotir, Thanas, Spiro, Sofika. Ils appartiennent tous au répertoire des prénoms chrétiens orthodoxes.

Les chrétiens les plus âgés ont tous des prénoms religieux et ont été baptisés jusque dans les années 1960. Les rituels du baptême et des fêtes patronales ont joué un rôle important pour la persistance plus tardive - en comparaison avec Fterra - de la transmission intergénérationnelle des prénoms. La concomitance de l'entrée dans la communauté religieuse et de l'obtention d'un prénom a profondément marqué la culture anthroponymique de l'espace orthodoxe. À Çorraj également, on trouve des prénoms albanais tels que Ilir (Illyrien) ou Jeta (vie) qui ont été attribués après 1970. Mais on continue de trouver certains cas de transmission de prénoms chrétiens.

Dans les deux villages, il semble que les pratiques de transmission intergénérationnelle des prénoms aient été beaucoup plus fréquentes auparavant. Les pratiques différentes d'attribution des prénoms dans le village chrétien de Çorraj, qui a transmis les prénoms chrétiens sur un mode patrilinéaire jusqu'à la fin des années 1960, et a repris cette tradition aujourd'hui, ne se distinguent pas fondamentalement des pratiques traditionnelles d'attribution des prénoms à Fterra. Toutefois, dans l'espace musulman, nous devons reporter notre attention du prénom au surnom. Car, dans l'islam, la filiation n'est pas traditionnellement exprimée par la transmission du prénom, mais par celle du surnom du fondateur de la lignée. Dans la culture chrétienne européenne, au contraire, ce sont les prénoms de saints et/ou d'ancêtres qui sont transmis ${ }^{25}$.

Ce qui distingue également les deux communautés religieuses et villageoises est la manière dont sont transmis les prénoms des vivants et des morts. Dans le Fterra musulman, on veillait non seulement à ce que le prénom n'apparaisse pas déjà dans le groupe de parenté, mais aussi à ce qu'il ne soit pas en usage dans la communauté villageoise. Le prénom d'une personne ne pouvait être utilisé que lorsque celle-ci était devenue très âgée, mais si quelqu'un mourrait prématurément, alors on évitait de transmettre son prénom. Tel n'est pas le

${ }^{25}$ Sur les théories concernant l'attribution "traditionnelle" des prénoms chrétiens et musulmans, voir Mitterauer (Michael), Ahnen und Heilige. Namengebung in der europäischen Geschichte, Munich : Beck, 1993, pp. 188, 427 ; Ahmed (Munir D.), Bürgel (Johann-Christoph) et alii, Der Islam, Band III (Islamische Kultur, zeitgenössische Strömungen, Volksfrömmigkeit), Stuttgart: Kohlhammer, 1990, p. 244 ; Mathewson (Denny F.), " Names and Naming ", in Eliade (Mircea), ed., The Encyclopedia of Religion, vol. 10, New York : Collier Macmillan, 1987, p. 305. 
cas à Çorraj, où étaient transmis les prénoms de parents vivants plus âgés. Toutefois, un seul enfant dans la parentèle pouvait hériter de ce prénom.

On peut supposer que cette différence trouve son origine dans différents modèles d'attribution des prénoms au sein des deux communautés. En milieu musulman, le prénom était avant tout lié à l'individu, avec l'existence concrète de la personne, car le prénom n'était pas renforcé par un sacrement religieux. En milieu chrétien, le prénom se rapportait à un saint à qui l'on s'adressait à travers l'individu, le prénom continuant de la sorte à dépasser celui-ci.

Il y avait toutefois un point commun entre les deux communautés : on évitait toute homonymie au sein de la mẽme génération. Pour ne pas provoquer le destin, on évitait aussi les homonymies au sein du village et de la parentèle. Comme il a été souligné plus haut, c'est seulement quand une personne était décédée que l'on pouvait redonner le prénom de la défunte ou du défunt. La tante de Monsieur X. Y. lui demanda de donner son prénom à ses enfants, car elle n'avait pas pu le transmettre à ses propres petites-filles : " elle était encore jeune, elle vivait encore et avait peur. Elle aurait été attristée si elle avait donné son nom à ses enfants ". La peur suscitée par la mort d'une personne et la stigmatisation de son prénom se retrouve à Fterra comme à Çorraj, y compris parmi les jeunes générations. Monsieur M. D., né en 1956, s'appelait Asqeri jusqu'à ce qu' un autre Asqeri meure dans un accident et que sa mère lui donne le nouveau prénom de (L)Ivan. Lefteri et Mariana, nées en 1950 et 1965 , ont changé leur prénom usuel pour Luisa et Voja, parce qu'une Lefteri etait morte dans le village et qu'une autre Mariana avait habité dans la même partie du village. Le prénom fut changé par égard envers le chagrin des personnes habitant à proximité, mais aussi parce que " ce r'est pas bon quand quelqu'un meurt et que l'on porte son prénom ".

Les gens de Fterra se rappellent encore aujourd'hui d'autres traditions qui associent le prénom à la mort. Monsieur V. W. parle de la " psychose des anciens " qui faisait que, auparavant, quand trois enfants décédaient à la suite dans une même famille, l'enfant suivant recevait un prénom chrétien. Par ce biais se manifestait l'espoir que le recours à un autre pouvoir divin permettrait de trouver un possible allié contre la mort ${ }^{26}$.

La croyance dans le pouvoir magique des prénoms était explicite dans certaines coutumes anciennes. Quand un enfant mourrait, le hoxha devait chuchoter à l'oreille du nouveau-né suivant son "véritable" prénom et ne rendre public que son prénom usuel de substitution. Cela devait le protéger des des-

\footnotetext{
${ }^{26}$ Les tabous sur les prénoms, qui sont très fréquents dans les pratiques culturelles anciennes mais aussi contemporaines, reproduisent sur un plan idèalisé la signification des prénoms dans la vie sociale. Sur les traditions anciennes qui associent le prénom à des éléments de pouvoir surnaturels, voir Mitterauer (Michael), op.cit. Sur les croyances albanaises et arvanites en particulier, voir Haussig (Hans Wilhelm), (Hg.), Wörterbuch der Mythologie - Band II (Götter und Mythen im alten Europa), Stuttgart : Klett-Cotta, 1973, p. 472 ; Jochalas (Titos), Arvanitika paramythia ke doxassies (Contes et croyances populaires arvanites), Athènes : T. Jochalas, 1997, p. 178.
} 
seins maléfiques d'individus ou de démons maîtrisant certaines pratiques magiques. S'ils invoquaient le faux prénom, ils ne pourraient pas utiliser leurs forces chtoniques contre le nouveau-né. Madame T. Q. raconte que, alors qu'elle était encore un petit enfant, ses parents modifièrent son prénom public afin que l'enfant suivant soit de sexe masculin. La magie des prénoms semble avoir été tout à fait "efficace", puisque l'enfant suivant de la famille fut un garçon!

À l'époque communiste, il n'y avait pas dans chaque famille et pour chaque enfant un rituel précis d'attribution du prénom. Si la famille était prospère, on avait l'habitude d'organiser une fête pour le fils, appelée davetë. Sinon, le prénom était simplement attribué au nouveau-né.

À l'époque communiste, le rituel du baptême chrétien n'a plus été pratiqué à partir de 1960 au plus tard. Les prénoms étaient librement choisis par le cercle restreint de la famille. À Fterra, la coutume du tirage au sort (alb. : me shorti) du prénom était largement répandue. Les parents ou plusieurs personnes proches de la famille se réunissaient, mettaient dans un chapeau de petits bouts de papier avec les prénoms proposés et tiraient l'un d'entre eux au hasard. Un modeste banquet s'en suivait. Le tirage au sort est interprété par les informantes et les informants comme une action qui facilitait le choix du prénom dans une large palette et permettait d'éviter les conflits entre les personnes concernées. Cette tradition est aussi liée au choix du prénom en fonction des rêves - on donne le prénom Flora parce que l'on a rêvé de flori (florins, monnaies d'or). Le fait qu'un signe surnaturel (tirage au sort, rêve prémonitoire, etc.) était attendu pour choisir le prénom confirme la croyance selon laquelle ce dernier a une influence sur la vie de l'enfant.

La signification historique du prénom était aussi très forte, bien qu'elle ne soit pas ancrée dans les règles contraignantes d'attribution des prénoms. On peut supposer que, dans les années 1970 également, malgré le primat des prénoms "nationaux", les prénoms étaient choisis en fonction de leur signification : Maylinda (Née en Mai), Liri (Liberté), Kastriot (nom d'un héros national), Mjaftone ("Cela suffit ${ }^{27}$ ) sont des prénoms qui renvoient à des dates anniversaire, à des significations, à des modèles héroïques albanais et à des voeux.

Aujourd'hui coexistent dans certaines familles des prénoms musulmans et des prénoms de stars de la télévision grecque. Les prénoms issus de la production cinématographique hollywoodienne sont également de plus en plus populaires. " Il n'existe aucune règle concernant la manière dont les noms doivent être transmis. Chacun peut faire une proposition. Nous avons beaucoup de beaux noms nouveaux, la plupart viennent de films ", dit un habitant de Fterra. Dans l'émigration, les nouveaux prénoms grecs sont généralement choisis en fonction de leur ressemblance avec l'ancien prénom albanais ou, chez les nouveaux-nés, sont proposés par les parrains et les marraines. Les mo-

${ }^{27}$ Ici, le septième enfant d'une famille. 
tifs et les modes de sélection des prénoms à l'étranger connaissent de fortes variations et ne nous permettent pas, dans le cadre de cette recherche, d'avancer des hypothèses sur les allitérations entre prénoms, les variations ou les éventuelles régularités dans l'attribution des prénoms.

\section{LE CHANGEMENT DE PRÉNOM : UN PONT ENTRE LE PASSÉ ET LE FUTUR ?}

L'évitement des homonymies et les traditions liées le plus souvent à une attitude particulière envers le surnaturel révèle certains aspects de l'univers symbolique des habitants. Dans les traditions de Fterra dont nous avons traité, il apparait que le prénom était et est encore lié à des superstitions concernant l'existence supposée de forces surnaturelles. Bien que les prénoms n'impliquent aucune attente particulière en ce qui concerne la vie après la mort, ils expriment une approche pragmatique de la mort au cours même de la vie terrestre. Dans ce contexte, le changement de prénom était tout à fait courant. De même, il était légitime de se servir de ce changement pour s'adresser à un autre pouvoir divin.

Cette approche pragmatique du prénom et de la religion, telle qu'elle apparaît dans les traditions, rappelle les déclarations des informantes et des informants sur leur "conversion" actuelle au christianisme. Il ne faut pas négliger le fait que beaucoup peuvent se décider à passer au christianisme par ce qu'ils sont convaincus qu'" il n'y a qu'un seul Dieu ". Le concept du divin et du sacré a pour beaucoup d'Albanaises et d'Albanais une signification qui dépasse les appartenances confessionnelles ${ }^{28}$. Avant tout, la foi doit agir sur le quotidien et apporter aux individus un soutien pour la vie ici-bas. Tant l'appartenance à l'islam que le passage actuel au christianisme s'expliquent par des considérations sociales.

De ce point de vue, les processus actuels de changement de prénom et de religion ne doivent pas être considérés comme une rupture avec les modèles identitaires traditionnels. Bien au contraire, les fonctions historiques de l'attribution des prénoms ont aujourd'hui rendu possible l'émergence d'identifications "mobiles" et facilité l'adaptation à un univers étranger. Les nouvelles valeurs des migrants se déploient en même temps que la capacité de surmonter l'adversité : la fierté tirée de sa propre flexibilité et de sa capacité à être justement dans le même temps Albanaise et Grecque, Albanais et Grec. Le switching of identities [29] peut ainsi être perçu comme un jeu de manœuvres habile qui met le prénom ainsi que la religion au service des pratiques quotidiennes.

\footnotetext{
${ }^{28}$ Lakshman-Lepain (Rajwantee), "Religions between Tradition and Pluralism ", Human Rights without Frontiers, 8 (2-3), 1996, pp. 10-11.

${ }^{29}$ N.d.T. : en anglais dans le texte (switching of identities : basculement des identités).
} 